\title{
Coulisses
}

Revue de théâtre

43 | Automne 2011

Reviviscences de Faust

\section{Low cost, ou le Faust de tout le monde}

\section{Peter Szendy}

\section{OpenEdition}

\section{Journals}

Édition électronique

URL : https://journals.openedition.org/coulisses/220

DOI : $10.4000 /$ coulisses. 220

ISSN : 2546-9460

\section{Éditeur}

Presses universitaires de Franche-Comté

\section{Édition imprimée}

Date de publication : 31 décembre 2011

Pagination : $37-47$

ISBN : 978-2-84867-404-9

ISSN : 1150-594X

\section{Référence électronique}

Peter Szendy, "Low cost, ou le Faust de tout le monde », Coulisses [En ligne], 43 | Automne 2011, mis en ligne le 30 novembre 2016, consulté le 29 décembre 2022. URL : http://journals.openedition.org/ coulisses/220 ; DOI : https://doi.org/10.4000/coulisses.220 


\title{
Low cost, ou le Faust de tout le monde
}

\author{
Peter Szendy
}

\author{
« LUST. - [...] vous ressemblez à tout le monde. \\ MÉPHISTOPHÉLÈs. - Prenez garde... \\ Rien n'est plus dangereux que tout le monde. \\ Écoutez donc un peu ce que tout le monde dit de \\ tout le monde...» \\ (Paul Valéry, Lust, I, 3)
}

1 Moi aussi, comme tant d'autres, comme tout le monde sans doute, $\mathrm{j}$ 'aurais aimé pouvoir dire : voici, ceci est mon Faust. Comme d'innombrables lecteurs avant moi, j'aurais voulu pouvoir user de ce syntagme, me le prononcer au moins à moi-même, car il n'est autre que la formule attestant d'une lecture.

«Mon Faust » : si je pouvais en effet énoncer ces mots, on saurait tout au moins qu'une lecture a bien eu lieu et que ce fut la mienne. Elle vaudrait ce qu'elle vaudrait, elle pourrait être une simple lecture parmi tant d'autres ou une réécriture fabuleuse, toujours est-il qu'elle porterait ma marque (ne serait-ce que dans ma mémoire de lecteur), elle garderait les traces de mon passage (même silencieux, discret, à moi seul connu) à travers le texte du mythe, à travers les archives de tous les Faust qui, à leur tour et tour à tour, se seront lus entre eux.

Or, «Mon Faust », on le sait, c'est le titre général sous lequel Paul Valéry a regroupé ces "ébauches» que forment Lust, une "comédie», et Le Solitaire, une "féérie dramatique $»^{1}$. Si bien que quiconque oserait aujourd'hui dire: voici, ceci est mon Faust ; quiconque prétendrait ainsi avoir lu Faust, d'une lecture digne de ce nom et qui soit donc proprement sienne, se trouverait déjà en train de citer le geste d'appropriation valéryen. Difficile d'imaginer plus cinglante dépossession : à l'instant même où je revendiquerais la Chose faustienne comme mienne - cette Chose, oui, qui n'est peut-être rien d'autre que cette revendication même -, elle s'avérerait sienne. Ou mieux : la forme de sa mienneté serait d'être sienne. 
4 Mais la dépossession, quand il s'agit de Faust, ne s'arrête pas là. Car Valéry, lui qui aura signé ce syntagme - «Mon Faust » - en en faisant sa Chose et en nous la soustrayant d'avance, Valéry lui-même aura dû le faire entre guillemets ${ }^{2}$, comme s'il devait s'en défaire aussitôt : "Mon Faust ", semble-t-il dire, n'est "mien » que pour ainsi dire. Et tout se passe dès lors comme s'il prédisait à chaque lecteur faustien à venir que Faust n'est et ne sera que faussement tien, qu'il vaut donc sans doute mieux s'en défausser, mais oui, tiens! je te le refile, nous dit-il, ce Faust qui te fausse faustiennement compagnie dès que tu le dis tien. pressés qui tendons à deviner, comme l'écrit Valéry en 1915, plutôt qu'à nous donner la peine d'une lecture digne de ce nom ${ }^{3}$ ?

7 Sur le seuil de son "Mon Faust ", nous sommes déjà comme voués à faire l'expérience, en le lisant, d'une expropriation radicale qui se loge au cœur même de sa propriété : à chaque fois que je lirai son "Mon Faust », à mesure que son "Mon Faust » deviendra un peu plus mon «Mon Faust », il redeviendra sien pour cette raison même, il m'échappera en lui revenant par le mouvement même qui le fera mien... Telle est l'abyssale logique du défaussement qui pointe dans l'entrebâillement des guillemets du titre.

personnage du docteur Faust - est synonyme d'un épuisement qui affecte d'avance tout ce qu'il pourrait s'approprier, c'est-à-dire tout. Comme le dit Méphistophélès dans la première partie de la tragédie de Goethe ${ }^{4}$ :

Le destin a donné à cet homme un esprit / Qui va toujours frénétiquement de l'avant, / Et dont l'élan précipité / Aura bientôt sauté par-dessus toutes les joies de la terre!

(Ihm hat das Schicksal einen Geist gegeben, / Der ungebändigt immer vorwärts dringt, / Und dessen übereiltes Streben / Der Erde Freuden überspringt.)

Lire Faust, ce sera donc suivre Faust dans la vitesse excessive d'une consumation ou consommation générale qui, de Goethe à Valéry, devient de plus en plus clairement synonyme d'une « mise en exploitation du globe » que nous nommerions aujourd'hui : mondialisation ${ }^{5}$.

Mais, dans la mesure où la lecture elle-même n'échappe pas à ce processus consumant (elle en participe plutôt qu'elle ne le constate), lire Faust, ce sera aussi, en tant que lecteur, faire l'épreuve de cette exhaustion qui inscrit d'avance, et à plus d'un titre, le signe d'une expropriation radicale au cœur de l'appropriation la plus déchaînée.

Goethe avait suivi de près les premiers vols en montgolfière, assistant par exemple, dans son propre jardin, à un essai réussi en juin 1784. Et il s'était fait l'écho, dans ses Maximes et réflexions (\$ 402), de "l'émotion universelle » suscitée par cette invention. Weltbewegung, disait-il : ce que l'on peut aussi entendre, d'une autre oreille et dans un sens à peine déplacé, comme un mouvement mondial ou mondialisant, à l'instar de

Coulisses, 43 | Automne 2011 
celui qui porte la Weltliteratur, cette « littérature mondiale » dont Goethe avait forgé le concept $^{6}$.

Dans le Premier Faust, la lecture elle-même est d'emblée placée sous le signe de l'envol et du survol, lorsque Faust, au cours du long monologue nocturne qui suit immédiatement le prologue dans le ciel, apostrophe la lune (v. 386 sq., je souligne) :

Ô, pleine clarté de la lune, si tu jetais / Pour la dernière fois un regard sur ma peine, / Toi que j'ai si souvent, à minuit, par mes veilles / Fait venir à ce pupitre : / Car par-dessus cet amas de livres et de papiers (über Büchern und Papier) / Mélancolique amie, tu m'apparaissais!/ Ah! Si seulement je pouvais parcourir les hautes montagnes / À ta douce clarté, / Planer avec les esprits (mit Geistern schweben) à l'orée des cavernes, / Flotter (weben) sur les prairies à ta pâle lumière...

Tout se passe donc comme si c'était la lecture elle-même qui, en décollant de la myope proximité des lignes sur la page, était appelée à devenir planante ou flottante ${ }^{7}$, suspendue dans ce surplomb qui serait aussi, nous y viendrons, l'élément du dé-lire inhérent à la figure d'un lecteur devenu lui-même faustien.

De fait, c'est d'un lecteur endiablé, c'est d'un lecteur porté à la précipitation et à l'épuisement rapide que parle le directeur du théâtre dans le prologue du Premier Faust de Goethe: "ils ont lu une effroyable quantité de livres", dit-il à propos des spectateurs qui ont déjà pris place pour assister à la tragédie (v. 46, sie haben schrecklich viel gelesen), et «nombre d'entre eux viennent après avoir lu la presse » (v.116, gar mancher kommt vom Lesen der Journale). Si bien qu'ils ressemblent déjà aux lecteurs modernes, aux lecteurs actuels évoqués par Valéry dans nombre d'entrées de ses Cahiers: " des gens qui ne peuvent pas me donner une quantité de temps et qualité d'attention comparable à ceux que je leur donne ", des gens qui, voués à la rapidité et au style télégraphique des journaux, ne font que « deviner au lieu de lire $»^{8}$.

Ce qu'il faudrait donc penser, et sans se dépêcher d'en juger, ce serait une alliance, une collusion, une sorte d'entente tacite entre, d'une part, le point de vue surplombant de la Weltbewegung et, d'autre part, le régime précipité du lire (ou du dé-lire) divinatoire qui aurait peu à peu émergé de la mondialisation littéraire, de la Weltliteratur.

Faust vole, on le sait, dans le premier volet de sa tragédie : «Il suffit d'étendre ce manteau, / Il nous portera à travers les airs ", lui dit Méphistophélès au vers 2065, en expliquant qu' « un peu d'air inflammable » (ein bisschen Feuerluft) les aidera à s'élever de cette terre.

Mais c'est dans le Second Faust que le regard aérien, que la vue d'en haut finit par déployer sa véritable portée cosmopolitique. L'acte quatrième (Haute montagne) s'ouvre ainsi sur les "cimes de rochers abrupts et dentelés ", dans un décor qui évoque d'avance le " toit du monde » où se jouera Le Solitaire de Valéry9. « Est-ce que rien ne t'a plu sur notre surface ? ", demande Méphistophélès (v. 10129) à Faust qui vient de sortir d'un nuage, avant de paraphraser les évangiles: "Tu as embrassé du regard ( $d u$ übersahst), dans des lointains sans borne, / Les royaumes du monde et leur gloire ${ }^{10}$. Plus loin, à l'acte cinquième, au seuil de la tombe que lui creuseront les lémures, sur le point de devenir aveugle, Faust déclare (v.11441): «Le cercle de la terre m'est suffisamment connu. » Et si, entre-temps, ce même "cercle terrestre » (Erdenkreis) lui semble "offr[ir] encore de l'espace aux grandes actions" (v. 10181), c'est dans la 
perspective de parachever son «empire [qui] est infini » (v. 11153) : tandis qu'affluent les "produits de régions du monde étrangères " (Erzeugnissen fremder Weltgegenden, v. $11165 \mathrm{sq}$.), il ordonne la destruction du dernier lopin de terre non conquise ni exploitée, à savoir le petit jardin de Philémon et Baucis sur la dune, préparant ainsi la revanche des éléments, l'entropique "anéantissement" (Vernichtung) qui réjouit d'avance Méphistophélès (v. 11550).

Ce qu'il importe de souligner ici, c'est que le terme de l'appropriation globale du globe terrestre entreprise depuis le point de vue surplombant du survol, la fin de cette prise de terres s'achevant dans l'épuisement coïncide avec la mort de Faust ${ }^{11}$. Mais cette mort, à son tour, c'est l'instant tant attendu où Faust appartiendra à Méphistophélès. Il deviendra enfin sa propriété à lui, qui pourra dès lors dire lui aussi, au bout du compte : «Mon Faust ».

Ou plutôt : il pourrait le dire, oui, si la mort était toujours ce qu'elle était, elle qui doit en quelque sorte faire le deuil d'elle-même, comme si un certain régime du mourir était lui-même en train de mourir. Parlant de l'âme, Méphistophélès déclare en effet (v. 11622 sq.) :

On ne peut plus se fier à rien (man kann auf gar nichts mehr vertrauen). / Autrefois elle s'échappait avec le dernier soupir, / Je la guettais et, comme la souris la plus rapide, / Hop ! Je la tenais dans mes griffes solidement fermées. / Maintenant, elle hésite et, cet endroit sinistre, / La demeure nauséabonde du cadavre gâté, elle ne veut plus les quitter ; / [...] La mort ancienne a perdu sa force rapide, / Même de savoir si reste longtemps une question douteuse ; / Souvent j'ai regardé avec convoitise les membres raides : / Ce n'était qu'apparence, cela bougeait, cela remuait à nouveau.

L'expropriation générale - revers de cet avers qu'est la mise en exploitation totale prend donc la forme extrême d'une crise de confiance qui touche la mort même.

Se pourrait-il que ce discrédit thanatologique qui dépossède Méphistophélès au moment même où il s'apprête à entrer en possession de son Faust, se pourrait-il que cette déstabilisation de la crédibilité du point final scellant le pacte consonne avec ce qui s'annonce comme une banqueroute du lecteur, condamné à ne pouvoir dire «mon Faust » qu'entre guillemets?

19 Faust, synonyme de la saisie globalisante du monde comme tout, apparaît aussi comme le nom de cette Chose qui, n'étant à personne, est finalement à tout le monde. Voilà ce dont le lecteur des trois Faust non seulement prendrait peu à peu connaissance, mais aussi ce dont il ferait, dans sa lecture même, l'expérience.

Au fil des ébauches pour son "Mon Faust ", Valéry accélère et amplifie la Weltbewegung goethéenne. Déjà, ce Troisième Faust, comme il l'appelle, est un Faust de l'ère de la conquête des airs. Il est vrai qu'il n'y a guère d'allusions au vol dans les scènes achevées, mais on peut lire par exemple sur tel feuillet d'esquisse ${ }^{12}$ :

Faust est le personnage européen par excellence. / Le voici qui reparaît au bout de cent ans. L'heure est excellente. / Toute une mythologie réalisée. Hommes volants, Centaures d'acier...

Si l'époque de ce nouveau Faust est donc plus que jamais celle des survols, elle voit également la concrétisation, certes balbutiante et le plus souvent risible, de l'idée kantienne d'une Société des nations ${ }^{13}$. Celle-ci est présente dans plus d'une note de Valéry pour son "Mon Faust », en s'y entrelaçant avec un autre motif récurrent et 
souvent commenté : la dévaluation des valeurs. Tout se passe dès lors comme si, dans ce Troisième Faust, les choses, vues d'en haut et depuis un point de vue cosmopolitique surplombant, apparaissaient de plus en plus égales, dans une indifférence ou équivalence générale ; ainsi peut-on lire dans telle entrée des Cahiers ${ }^{14}$ :

Un III ${ }^{\mathrm{me}}$ Faust / Toute magie dépréciée - [...] Vulgarisation des puissances et des prestiges. Le nigaud vole. La sottise chevauche la lumière. / [...] Nations - Société des Nations - À Genève [*]. / Méphistoph[élès] en noir pur. / Toujours en retard - / La vitesse - / Nature empoisonnée.

[*ajout:] Séance où par la sorcellerie de Méph[istophélès] ils disent tout à coup la vérité - l'arrière-pensée. Mais [phrase inachevée.]

Les ascensions qui, chez Goethe, étaient propulsées par l'air inflammable (Feuerluft), ces décollages sont devenus des envolées dévaluées, des vols low cost. En faisant circuler Faust toujours plus vite et toujours plus souvent autour du globe, en lui faisant faire et refaire, comme à tout le monde, le tour du monde, ce survol à bon marché le place pour ainsi dire sur l'orbite d'une version rognée, rongeuse et corrodée de l'éternel retour, que les Cahiers nomment « cyclose ", comme s'il s'agissait d'une maladie ${ }^{15}$ :

Je fus frappé et exaspéré de fort bonne heure par la nature périodique de la " vie »dans son cadre d'orbites, de saisons - de redites [...]. « Mon Faust » est l'homme qui a trop conscience de cette cyclose et cette conscience lui remet tout le temps le nez dans cette cyclomanie de notre essence.

Ça tourne et ça retourne, jusqu'au vertige : tout le monde circule cyclomaniaquement autour du monde comme tout. Et c'est depuis cette pathologie circulaire de la circulation générale accélérée qu'émerge l'affect du bouleversant ennui, tel qu'on peut l'entendre lorsque Faust s'adresse au Disciple (Lust, II, ${ }^{16}$ ):

J'ai fait le véritable tour du véritable monde... Puis, toujours entraîné par ma fatalité, je revins dans le temps... Je vins revivre. Je revis. Je vis, je vois, je connais, si c'est vivre, voir et connaître que de revivre, de revoir et de reconnaître. [...] L'idée la plus rare et la plus hardie qui me vienne ne me donne jamais plus l'impression d'une nouveauté. Il me semble, aussitôt surgie, l'avoir déjà pensée et repensée...

L'ennui était certes déjà présent chez Goethe : tel spectateur de la tragédie, disait le directeur du théâtre dans le prologue du Premier Faust, "vient poussé par l'ennui » (diesen Langeweile treibt, v.113), tandis que, dans le Second Faust, Méphistophélès s'ennuie déjà (mich langeweilt's, « ça m'ennuie », v. 6958) sur le seuil du voyage en ballon qui le fera arriver à la Nuit de Walpurgis classique en ayant survolé à rebours, vers le passé, tout un pan de la bibliothèque mondiale.

Mais jamais l'ennui, chez Goethe, ne gagne encore tout le monde et le tout du monde, comme il le fera en revanche dans le Troisième Faust, lors de l'inoubliable conversation blasée entre les deux diables que sont Astaroth et Bélial, figures du monnayage universel et de la salissure générale :

ASTAROTH. - Je m'ennuie, je m'ennuie... Oh! que je m'ennuie !... Krèk, krèk. [...] Je ronge, je rogne, je lime, j'effrite... Tout m'ennuie, l'ennui me ronge... Krèk, krèk... BÉLIAL. - Oh ! ce crin, crin... Qu'est-ce que tu ronges ? ASTAROTH. - Tout... Les cœurs, les corps, les gloires, les races, les roches, - le Temps lui-même... Je mets en poudre... Krèk, krèk... BéLIAL. - Chacun son goût... Tu ronges, moi, je souille !... ASTAROTH. - Pouah! Et qu'est-ce que tu souilles? BÉLIAL. - Tout. (Lust, III, 1 ; ibid.,

p. 332-333)

Cette érosion entropique (qui a tant à voir avec ce que Nietzsche décrivait comme un devenir-sable ${ }^{17}$ ), c'est l'effritement qui accompagne comme son ombre la globalisation de la Weltbewegung. Mais pareille dislocation s'annonce aussi, simultanément, au sein du mouvement totalisant de la Weltliteratur. Non seulement parce que celle-ci décrit, représente, peint ou donne à voir ce que le Troisième Faust, dans la lignée des deux 
premiers, nomme le "véritable tour du véritable monde ». Mais aussi parce qu'elle accomplit, dans l'élément même du langage, une "panthèse ", comme dit joliment Valéry : «Idées p[our] Faust III - Théanthropomythologie ou Panthèse ", lit-on dans une entrée des Cahiers (1940, II, p. 1344), comme si la langue elle-même était entrainée dans un mouvement d'accumulation universelle où le texte faustien tendrait à la limite à devenir un unique et vaste mot-valise, à se gonfler en un signifiant monstre ("théanthropomythologie ») qui se craquèle néanmoins ("théo »), qui se fragmente ("anthropo »), se désagrège ("mytho») dans le mouvement même du logos qui le totalise (« logie »).

De fait, nous y venons, avec le sablage corrosif de tout le monde, ce qui guette, c'est aussi la ruine dans le commerce avec les signes.

Une banqueroute, disions-nous, de et dans la lecture. frankensteinienne de l'homunculus qui apparait à l'acte II. Ce petit homme artificiel, obtenu non par «la procréation à l'ancienne mode » (laquelle "n'était qu'une farce ", v. 6839) mais «à partir de plusieurs centaines de matières, par mélange » (v. 6850), cet humanoïde accompagne Méphistophélès et Faust endormi dans le voyage en ballon qui les fait arriver, après avoir surplombé des siècles et des siècles de littérature universelle, à la Nuit de Walpurgis classique. Volant avec Faust et Méphistophélès, planant un peu au-dessus d'eux, il est comme leur lampe de chevet aérostatique : « Quel météore inattendu ! / Cela brille et éclaire un corps en forme de ballon », s'exclame la magicienne Érichtho en voyant l'étrange liseuse flotter dans les airs (v. 7035). Et l'on comprend sa surprise, à ce personnage littéraire qui fut évoqué par Ovide comme par Dante ; on comprend la stupéfaction d'Érichtho, depuis ce qui semble être son point de vue à ras du sol (ou mieux : à ras du texte qu'elle habite), lorsqu'elle aperçoit au-dessus d'elle l'improbable dispositif de lecture qui est en train de la lire au vol...

L'homunculus, dans cette scène plus fantastique encore que toutes les autres, est comme un rayon de lumière qui scanne à perte de vue l'archive des belles lettres («je parcourrai un petit morceau de monde », ein Stückchen Welt, dit-il au vers 6993), en faisant remonter le temps de la lecture aux lecteurs volages qu'il transporte dans l'atmosphère. Mais ce n'est pas seulement vers le passé qu'il les fait s'envoler, lui qui est aussi l'incarnation de l'anticipation inhérente à l'acte de lire : lévitant au-dessus de Faust et l'éclairant (schwebt über Faust und beleuchtet ihn, v. 6903), il entrevoit ce que celui-ci songe ; si bien qu'il se téléporte ainsi d'avance, en " grand visionnaire » qu'il est (v. 6922), vers ce que Faust ne vivra que plus tard avec un sentiment de déjà-vu ${ }^{18}$. C'est ainsi, en somme, que l'homunculus décolle vers le passé ou l'avenir de la lecture, en un mouvement dont le paradigme est plus que jamais la circumnavigation aérienne.

Or, cette tête de lecture androïde est une figure du lecteur qui reste absolument indécidable. D'une part, en effet, il est l'hyperlecteur, devin ou prophète en même temps que dépositaire de l'histoire de la littérature mondiale, hypermnésique autant qu'hypervoyant. Mais d'autre part, il est aussi comme l'allégorie d'un lecteur cloné, artificiellement reproduit, machinal ou machinique. Bref, il est également celui que Valéry rend responsable de l'agonie de la littérature, elle qui se meurt d'être lue à coups de divinations et de survols surplombants : 
L'évolution de la littérature moderne n'est que l'évolution de la lecture qui tend à devenir une sorte de divination d'effets au moyen de quelques mots vus presque simultanément et au détriment du dessin des phrases. / C'est le télégraphisme et l'impressionnisme grossier dû aux affiches et aux journaux. L'homme voit et ne lit plus. / [...] En somme la littérature veut essayer de survivre aux conditions de la littérature, - qui sont lecture et science de la lecture; culture; connaissance des mots et des formes, poids des significations; nuances - Toutes choses moribondes. (Cahiers, 1918-1919, II, p. 1183)

Chez Valéry, c'est donc chacun, c'est tout le monde qui tend à devenir l'homunculus, c'està-dire le lecteur volage, lisant au vol et à une allure que Goethe qualifierait peut-être de vélociférienne ${ }^{19}$. La lecture survolante et surplombante, avec ses vertus divinatoires et ses transports globalisants, s'en trouve évidemment dévaluée, même si elle reste pourtant le modèle sur lequel se règle implicitement le livre absolu, l'hyperlivre que Faust rêve d'écrire :

FAUST. - Écoute : Je veux faire une grande œuvre, un livre... MÉPHISTOPHÉLÈs. - Toi ? Il ne te suffit pas d'être toi-même un livre ?... FAUST. - J'ai mes raisons. Il serait un mélange intime de mes vrais et de mes faux souvenirs, de mes idées, de mes prévisions, d'hypothèses et de déductions bien conduites, d'expériences imaginaires: toutes mes voix diverses! On pourra le prendre en tout point, le laisser en tout autre... MÉPHISTOPHÉLÈs. - Ceci n'est pas trop neuf. Chaque lecteur s'en charge. FAUST. - Personne, peut-être, ne le lira ; mais celui qui l'aura lu n'en pourra plus lire d'autre. MÉPHISTOPHÉLÈs. - Il sera mort d'ennui... FAUST. - [...] J'ai donc ce grand ouvrage en tête, qui doit finalement me débarrasser tout à fait de moi-même, duquel je suis déjà si détaché... (Lust, I, 2, p. 297-298)

Le livre de Faust, cet ouvrage dans lequel il se raconterait si bien qu'il pourrait enfin (se) dire (de) lui-même : je suis à moi, je suis mon Faust - ce livre serait aussi celui qui interdirait toute coïncidence de Faust avec soi («ce grand ouvrage [...] qui doit finalement me débarrasser tout à fait de moi-même »).

Ce "Mon Faust" serait le chef d'œuvre de chaque lecteur ("chaque lecteur s'en charge $»^{20}$ ) - à condition d'être indifférent à ses lectures (« on pourra le prendre en tout point, le laisser en tout autre »).

Ce livre de tous les Faust serait le livre des livres (« qui l'aura lu n'en pourra plus lire d'autre ») - mais qui ne vaudra pas plus que tous les autres (« qui l'aura lu [...] sera mort d'ennui »).

Et face à Méphistophélès, dépossédé comme tout le monde ${ }^{21}$, face à ce représentant d'un Diable en faillite qui parle également pour le lecteur quelconque ("chaque lecteur»), ce livre serait enfin, en un volume, sous une seule et même couverture, la Weltiteratur, la littérature mondiale devenue la littérature de tout le monde.

\section{NOTES}

1. « Mon Faust » (Ébauches), in CEuvres, II, Gallimard, « Bibliothèque de la Pléiade », 1960, p. 276 sq.

2. Sur les guillemets, $c f$.telle entrée des Cahiers de Valéry en 1935 (tome I, Gallimard, «Bibliothèque de la Pléiade », 1973, p. 282) : «Je mets entre guillemets comme p[our] mettre, non tant en évidence, qu'en accusation - C'est un suspect. Ou bien je suppose au sens l'idée de l'emploi 
qu'en font tels ou tels. Je ne prends pas la responsabilité - du terme - etc. / Guillemets = provisoire. » - Jean-Michel Rey parle très justement de «l'idée évoquée dans "Mon Faust " : qu'un titre de livre puisse être volé avant d'avoir été produit » (Paul Valéry. L'aventure d'une œuvre, Seuil, "La Librairie du Xx ${ }^{\mathrm{e}}$ siècle », 1991, p. 29).

3. Cahiers, tome II, Gallimard, «Bibliothèque de la Pléiade », 1974, p. 1165 : « Le grand problème de l'écrivain moderne est de se faire lire, j'entends : d'empêcher le lecteur de deviner la phrase, la page. / On appelle obscur l'écrit qui ne livre son sens qu'à la lecture et non à simple vue. Il en sera ainsi de plus en plus. / Il faut obliger ce lecteur à l'exécution. » Je citerai désormais les entrées des Cahiers en donnant l'année, le tome et la page.

4. Faust I, v. 1854-1859. Je cite la traduction française de Jean Lacoste et Jacques Le Rider : Goethe, Faust. Urfaust, Faust I, Faust II, Bartillat, 2009.

5. Cf. la seconde des deux lettres qui composent «La crise de l'esprit », in Paul Valéry, Cuvres, I, Gallimard, «Bibliothèque de la Pléiade», 1957, p. 1000: "Le phénomène de la mise en exploitation du globe, le phénomène de l'égalisation des techniques et le phénomène démocratique, qui font prévoir une diminutio capitis de l'Europe, doivent-ils être pris comme décisions absolues du destin?». Quant à Faust, il déclare, dans l'intermède sur lequel s'interrompt Le Solitaire: "...j'ai plus que vécu, surmonté mainte crise, / Consumé tous les biens, tous les espoirs perdu » (« Mon Faust » (Ébauches), in CEuvres, II, op. cit., p. 398).

6. $C f$. les extraits traduits par Jean-Marie Schaeffer sous le titre de «Littérature universelle » in Goethe, Écrits sur l'art, Flammarion, 1996, p. 298-300. Dans la perspective qui nous intéresse ici, on notera en particulier que la question de la Weltliteratur croise celles, cosmopolitiques, de la «paix universelle » et de "ce qui appartient à toute l'humanité», mais aussi et surtout qu'elle se présente en termes d' « échange » et de « marché » (je souligne) : « Il faut apprendre à connaître les particularités de chaque nation, afin de les lui laisser, ce qui justement permet qu'on entre en échange avec elle : car les particularités d'une nation sont comme sa langue et sa monnaie, elles facilitent les échanges, davantage même, elles seules les rendent possibles dans toute leur ampleur. [...] Celui qui comprend et étudie la langue allemande s'installe sur un marché où toutes les nations font offre de leurs marchandises, il fait fonction d'interprète tout en s'enrichissant lui-même. [...] la traduction est et restera une des activités les plus importantes et les plus dignes dans l'échange mondial universel. "

7. Le retour du désir d'envol, aux vers 1074 sq., n'est pas loin d'évoquer également une lecture que l'on pourrait appeler «flottante» (schwebend, à l'instar de l'écoute théorisée par Freud), lorsque Faust parle de «tout un monde silencieux » se déroulant sous ses pieds (die stille Welt zu meinen Füßen).

8. 1924, I, p. 258-259. On trouve la même formule en 1916 : « Il y a la démocratie, l'affaiblissement des têtes, des disciplines de tout ordre, de la politesse d'écouter et du pouvoir de suivre. Les journaux. La rapidité. [...] Deviner au lieu de lire. Etc. Style télégraphique. » (II, p. 1169)

9. «Mon Faust " (Ébauches), in Euvres, II, op. cit., p. 381.

10. $C f$. Matthieu, 4, $8:$ : Le diable l'emmène encore sur une très haute montagne; il lui montre tous les royaumes du monde avec leur gloire... »

11. Saisissant aparté que celui de Méphistophélès au vers 11558 , où ce que Faust croit être le fossé (Graben) censé permettre de gagner des territoires sur la mer s'avère être en réalité sa fosse, sa tombe (Grab).

12. "Études pour "Mon Faust" ", f ${ }^{\circ} 37$, cité par Julia Peslier dans sa remarquable faustologie comparée: «Faust à l'épreuve du médiéval. Mémoires du Faust-Phénix chez Pessoa et Valéry, Boulgakov et Mann », Littérature, $\mathrm{n}^{\circ} 148,2007$, p. 91.

13. Sur le cosmopolitisme, le surplomb et la conquête mondialisante de l'espace, je me permets de renvoyer à Kant chez les extraterrestres. Philosofictions cosmopolitiques (Minuit, 2011).

14. 1930-1931, II, p. 1331-1332. - Dans sa thèse de doctorat en littérature comparée (La Pensée à l'œuvre. Chantiers de Faust, 2007, inédit), Julia Peslier cite le fo 165 des «Études pour “ Mon Faust " ", 
qui fait aussi allusion à un «Tableau de la Société des Nations » où « Méphistophélès fait dire la vérité par tous ".

15. 1943-1944, I, p. 313. Ailleurs, Valéry note aussi : «Faust III. [...] Je ferais un F[aust] en victime du Retour éternel; châtié d'avoir voulu recommencer - (1). / Ma $1^{\text {re }}$ idée - déjà vieille Reprendre le thème Faust pour le placer dans le Monde actuel - (2). / Si on combine (1) et (2) on trouve qu'il faut mettre en évidence l'accélération, caractère fatal du moderne. » (1940, II, p. 1345) 16. " Mon Faust » (Ébauches), in Euvres, II, op. cit., p. 312. - Cf. aussi la " Note sur "Mon Faust" ", citée in Cahiers, I, p. 1459: " "Mon Faust” est fort différent du Faust de Goethe. Il [...] semble, autant qu'on puisse le savoir, qu'il lui a été infligé pour châtiment de revivre. Il connaît la vie par cœur, et il lui faut la subir. Il ressent des événements et de tout, les mêmes dégoûts qu'un homme trouve dans son métier au bout de vingt ans qu'il le pratique. L'imprévu lui-même fait partie de ce prévu écœurant.» - Sur le bouleversant ennui comme affect de l'équivalence générale, cf. Tubes. La philosophie dans le juke-box, Minuit, 2008, passim.

17. On peut lire en effet, dans un fragment posthume de 1880 : «Plus le sentiment de leur unité avec leurs semblables prend le dessus chez les hommes, plus ils s'uniformisent, plus ils vont ressentir rigoureusement toute différence comme immorale. Ainsi apparaît nécessairement le sable de l'humanité : tous très semblables, très petits, très ronds... Jusqu'à présent ce sont le christianisme et la démocratie qui ont conduit l'humanité le plus loin sur la voie de cette métamorphose en sable."

18. "S'agit-il de rêves? Ou de souvenirs ? Une fois déjà, tu fus ainsi comblé de bonheur...» (v. 7275-7276)

19. Veloziferisch, mot-valise contractant Lucifer et la vitesse au $§ 180$ des Maximes et réflexions.

20. Cf. aussi telle entrée des Cahiers consacrée aux chefs-d'œuvre de lecture : « Chefs-d'œuvre / Ce n'est jamais son auteur qui fait un “chef-d'œuvre ". Le chef-d'œuvre est dû aux lecteurs, à la qualité du lecteur. Lecteur étroit, avec finesse, avec lenteur, avec le temps et la naïveté armée / Lui seul peut faire chef-d'œuvre, exiger la particularité, le soin, les effets inépuisables, la rigueur, l'élégance, la durée, la reprise. / mais ce lecteur, dont la formation et les fluctuations constitueraient le vrai sujet de l'histoire de la littérature, il se meurt... » (1916, II, p. 1167)

21. «Dessaisi », lui dit Faust, " parmi tous ces gens nouveaux qui pèchent sans le savoir » (Lust, I, 2, p. 303). Tant d'autres passages de Lust parlent du discrédit et de la paupérisation du Diable: "Écoute », lui lance Faust (ibid., p. 294-295), « je ne puis te cacher que tu ne tiens plus dans le monde la grande situation que tu occupais jadis. [...] Oh! je ne parle pas de ton chiffre d'affaires, ni même des bénéfices nets. Mais le crédit, la considération, les honneurs... » Ou encore (ibid., p. 302) : « tu n'inspires peut-être plus la même confiance. Il n'est pas écrit que l'on ne trouvera jamais quelqu'un de pire... [...] Tout le système dont tu étais l'une des pièces essentielles n'est plus que ruine et dissolution. »Sans doute ce dépôt de bilan de la diabolicité tient-il à ce qui s'annonçait déjà chez Goethe comme le deuil de la bonne vieille mort : " La mort », dit Faust (ibid., p. 302), « n'est plus qu'une des propriétés statistiques de cette affreuse matière vivante. Elle y perd sa dignité et sa signification... classique ». 
INDEX

oeuvrecitee Mon Faust

Keywords : Weltliteratur

Mots-clés : Weltliteratur

Palabras claves : Weltliteratur

AUTEURS

PETER SZENDY

Université de Paris Ouest-Nanterre-La Défense 